\title{
Konsumenten- und Agentenethik* \\ Zur Verantwortung für moralisches Handeln von und in Unternehmen
}

\author{
DominiK ENSTE, INNA KNELSEN UND MiCHAEL NEUMANN ${ }^{* *}$
}

Um trotz der wachsenden Komplexität wirtschaftliche Prozesse und ethische Verpflichtungen vereinen zu können, ist die Zuweisung von klaren Verantwortlichkeiten unumgänglich. Der vorliegende Beitrag diskutiert vor dem Hintergrund der PrinzipalAgenten-Theorie sowie der Empirie aus dem Ersten Engagementbericht, ob und inwieweit die Unternehmenseigner selbst und ihre Mitarbeiter im Vergleich zu den Konsumenten einen deutlich stärkeren Einfluss auf die Einhaltung moralischer Werte von und in Unternehmen leisten können und leisten sollten, da sie sowohl besser informiert sind als auch mehr Optionen haben, ethisches Verhalten einzufordern.

Schlagwörter: Konsumentenethik, Agentenethik, bürgerschaftliches Engagement, Prinzipal-Agenten-Theorie

\section{Consumer and Agent Ethics. About the accountability for moral action of and inside companies}

Societies are confronted with a growing complexity of economic processes. At the same time, and due to this, companies feel an increasing ethical responsibility. In order to combine market forces and moral action despite this trend, it is essential to allocate responsibilities very clearly. Based on the PrincipalAgent-Theory and empirical data from Germany's "first civic engagement report" this article discusses if and to what extent entrepreneurs, managers and employees can use their influence within the company to push moral concepts and behavior. They are better informed, have a wider range of opportunities and a bigher involvement than customers to establish ethical behavior in the company.

Keywords: Consumer Ethics, Agent Ethics, civic engagement, Principal-Agent-Theory

\footnotetext{
Beitrag eingereicht am 11.04.2012; nach doppelt verdecktem Gutachterverfahren überarbeitete Fassung angenommen am 03.07.2012.

** Dr. Dominik Enste, Kompetenzfeldleiter im Institut der deutschen Wirtschaft Köln, KonradAdenauer-Ufer 21, D-50668 Köln, E-Mail: enste@iwkoeln.de, Forschungsschwerpunkte: Institutionenökonomik, Verhaltensökonomik, Wirtschafts- und Unternehmensethik. Zugleich Professorenvertreter an der Fachhochschule Köln und Dozent an der Universität zu Köln und Geschäftsführer der Akademie für Integres Wirtschaften (IW Akademie GmbH).

Inna Knelsen, Junior Economist, Institut der deutschen Wirtschaft Köln, Konrad-Adenauer-Ufer 21, D-50668 Köln, E-Mail: knelsen@iwkoeln.de, Forschungsschwerpunkte: Verhaltensökonomik, Wirtschaftsethik.

Prof. Dr. Michael Neumann, Jade Universität, Friedrich-Paffrath-Straße 101, D-26389 Wilhelmshaven, Tel.: +49-(0)4421-985-2964, E-Mail: michael.neumann@jade-hs.de, Forschungsschwerpunkte: Volkswirtschaftslehre, Institutionenökonomik, Unternehmensethik.
} 


\section{1. $\quad$ Einleitung}

Unternehmen sind Institutionen mit dem Auftrag, dem Allgemeinwohl zu dienen. Wurde früher als Hauptziel eines jeden Unternehmens in Milton Friedmans Diktion auf die Profitmaximierung verwiesen, erwartet die moderne Gesellschaft von einem Unternehmen darüber hinaus mehr und mehr die Übernahme von Mitverantwortung im öffentlichen Raum. Das Spektrum der Verantwortung reicht hierbei von der Entwicklung ökologisch korrekter Produktionsprozesse bis hin zu konkreter Einflussnahme auf gesellschaftliche und soziale Probleme durch Spenden, bürgerschaftliches Engagement bis hin zur Verantwortung für die Teilnahme am gesellschaftlichen Diskurs.

Die Finanzkrise hat den Unmut der Gesellschaft über moralisch fragwürdige Handlungen in der Wirtschaft verstärkt, indem sie ihr schmerzlich die Konsequenzen solchen Handelns aufgezeigt hat. Das Bekanntwerden der hohen Boni-Zahlungen an gescheiterte Manager stieß nicht nur in weiten Teilen der Gesellschaft auf Unverständnis, sondern führte zudem zur Frage nach Systemänderungen und der Mitverantwortung für rechtzeitige, präventive Krisenverhinderung. Aufgrund der wachsenden Komplexität der Systeme und der zunehmenden Anonymität der Märkte erweist sich der Diskurs über die Verantwortlichkeiten als notwendig, um zu vermeiden, dass aus einer Finanzkrise über eine Staatsschuldenkrise letztlich eine Demokratiekrise wird.

Doch wie kann ein Unternehmen in diesem Kontext Verantwortung übernehmen und selbst moralisch handeln? Wer kann Druck auf jene Unternehmen ausüben, die nur unter Druck moralischen Anforderungen Genüge tun? Können Konsumenten diesen Druck ausüben? Wie lässt sich die Rahmenordnung im Sinne Homanns als Ort der Moral ergänzen? Welche diskretionären Handlungsspielräume bestehen für die im Unternehmen handelnden Akteure und wie lassen sich diese für die Implementierung moralischer Standards in Unternehmen nutzen? Welche Bedeutung haben in diesem Kontext Informationsasymmetrie und Prinzipal-Agenten-Theorie? Und wie beurteilen Unternehmen den Einfluss von Agenten und Konsumenten?

Der vorliegende Beitrag widmet sich diesen Fragestellungen vor dem Hintergrund, inwieweit welche Anspruchsgruppen im Unternehmen als primäre Träger der Mitverantwortung für gesellschaftliche Belange auszumachen sind. Der Beitrag rekurriert dabei auf die aus der Institutionenökonomik bekannte Prinzipal-Agenten-Theorie, die bei asymmetrischer Informationsverteilung dem Agenten den diskretionären Handlungsspielraum zuweist und ihn damit in der Ausbeutungsposition sieht. Es wird verdeutlicht, dass die Erwartungen an die Konsumenten teilweise zu hoch sind und mehr die Mitarbeiter als Agenten in den Fokus der Unternehmensethik genommen werden sollten. Erste Ergebnisse einer repräsentativen Befragung von rund 4.400 Unternehmen zeigen die Einschätzung der Unternehmen dazu. Kapitel 2 greift die Grundfragen der Unternehmensethik nach den Orten moralischen Handelns auf und ordnet so das Thema in die Ordnungsethik ein. Kapitel 3 diskutiert den Einfluss der Konsumenten, Kapitel 4 erörtert den Einfluss der Agenten. Ein Fazit mit Ausblick (Kapitel 5) rundet den Beitrag ab. 


\section{Unternehmensethik: Theoretische Grundlagen der Agentenethik}

Unternehmen sind ein essenzieller Bestandteil der freiheitlichen, marktwirtschaftlichen Ordnung. Sie sind verantwortlich für Bedürfnisbefriedigung und Produktion, Wachstum und Wohlstand, Innovation und Entwicklung (vgl. Erster Engagementbericht der Bundesregierung 2012). Kontinuierliche Anpassungen an Marktentwicklungen erweitern den Kundennutzen, sorgen für Gewinne auf der Unternehmensebene und für wachsenden Wohlstand in der Gesellschaft. Unternehmerisches Handeln dient damit dem Allgemeinwohl. Zumindest ist dies in der Regel der Fall - wenn die Rahmenordnung stimmig ist. Als systematischer Ort, Moral in einer Marktwirtschaft zu implementieren, gilt daher die gesellschaftliche Rahmenordnung (Homann/Blome-Drees 1992: 35). Die Regeln der Rahmenordnung lenken die Handlungen und das Verhalten der Gesellschaftsmitglieder, in der Politik wie auch an den Märkten, setzen Anreize für Individuen wie Unternehmen. Unter die Regeln fallen zum einen kulturelle Normen, Grundrechte und spezifische Gesetze, zum anderen aber auch Steuer- und Subventionsregelungen, welche Individuen wie Organisationen in ihren Zielen, Strategien und Prozessen beeinflussen (vgl. Göbel 2010: 107f.).

Staatliche Rahmenbedingungen sind im Hinblick auf die Lenkung von Handlungen und Verhalten indes unvollständig. Diese Unvollständigkeit lässt sich nicht zeitnah durch Änderungen der Regel- und Anreizsetzungen überwinden (Homann/BlomeDrees 1992). Die Unvollständigkeit der Regeln ist zumeist vom Regelsetzer ungewollt - es liegen asymmetrische Informationen zwischen Regelnehmer und Regelsetzer vor. Die verzögerte Reaktion der Gesetzgeber ist auf das Entstehen asymmetrischer Informationsverteilungen hinsichtlich moralischer Fehlanreize der Unternehmen zurückzuführen. Diese entstehen aus dem Zusammenspiel von alten und neuen Regeln oder technischen Entwicklungen. Auch können sie auf Kontrollprobleme zur Einhaltung der Gesetze zurückzuführen sein. Gesetze werden neuen Tatbeständen erst angepasst, wenn sie sich als so lückenhaft erwiesen haben, dass die gesellschaftlich unerwünschten Folgen zumindest von Teilen der Gesellschaft als drängend wahrgenommen werden. Mithin wird der Regelsetzer erst dann mit der Anpassung der Regeln die Fehlanreize korrigieren und den Handlungsspielraum der Unternehmen einengen, wenn die Informationsasymmetrie überwunden ist.

Als Folge dieser Lücken in der Rahmenordnung bestehen diskretionäre Handlungsspielräume für die Akteure der Wirtschaft, welche Entscheidungs- und Verhaltensfreiheiten eröffnen. Innerhalb dieses unvollständigen Regelrahmens verbleibt den Unternehmen ein Spielraum zur Lösung des Zielkonfliktes zwischen wirtschaftlich effizientem Handeln, das seinen Ausdruck im unternehmerischen Streben nach Gewinnen findet, und moralisch richtigem Verhalten, zum Beispiel fairen Arbeitsbedingungen oder dem Vermeiden von Umweltverschmutzung, findet. Das Unternehmen muss demnach in der Lage sein, die verschiedenen und oft gegensätzlichen Interessen seiner Stakeholder zu erkennen und diese unter wirtschaftlichen und moralischen Gesichtspunkten miteinander in Einklang zu bringen (vgl. Göbel 2010: 113). Als Ziel des Unternehmens gilt mithin die Herstellung einer Win-Win-Situation für die Organisation sowie für ihre unterschiedlichen Stakeholder. Eine solche Situation lässt sich aber nicht für alle möglichen Aktionsfelder erreichen, denn die Lücken in der Rahmenord- 
nung ermöglichen unmoralische Ausbeutungsstrategien, die, wenn Wirtschaftsakteure ihr Handeln allein vom Gewinnstreben leiten lassen, zulasten Dritter gehen können (vgl. Pies/Sardison 2005).

Durch den Wettbewerbsdruck unterliegt ein Unternehmen am Markt einem anonymen Zwang, seinen moralischen Standard an ein niedrigeres Niveau anzupassen (vgl. Steinmann/Löhr 1994: 27ff.): Agiert ein Unternehmen am Markt als Grenzmoralist, nimmt es die letzte moralisch vertretbare Position in dem ihm gegebenen diskretionären Handlungsspielraum ein. Durch den bewussten Verzicht auf Investitionen in Vertrauenskapital wird dem Grenzmoralisten eine Niedrigpreispolitik ermöglicht, die den Konsumentenwünschen entgegenkommt (vgl. Noll 2002: 49). Dies gilt zumindest dann, wenn die Kunden nicht bereit sind, für höhere moralische Standards einen höheren Preis zu zahlen. Die internationalen Dimensionen des heutigen Wirtschaftsraumes und die daraus resultierende Anonymität zwischen den Marktteilnehmern führen jedoch zu Informationsasymmetrien zwischen dem Konsumenten, den Unternehmen und den Konkurrenten des Grenzmoralisten. Ein Unternehmen, das entsprechende Kosten für einen höheren moralischen Standard in Kauf nimmt, wird vom Konsumenten deshalb nicht unmittelbar honoriert: Erstens muss das Unternehmen bei simultanem Effizienzstreben die Kosten an den Konsumenten weitergeben und daher das Preisniveau des Grenzmoralisten überschreiten. Zweitens ist der nach Nutzenmaximierung strebende Konsument nicht bereit diese Kosten zu tragen, soweit Informationsasymmetrien bestehen und er die Ursache des höheren Preises nicht nachvollziehen kann. Der Grenzmoralist ist somit aus Sicht des Kunden attraktiver. Folglich müssen sich konkurrierende Unternehmen an den Grenzmoralisten und dessen niedrigen ethischen Standard anpassen, wenn sie am Markt bestehen wollen - soweit beim Kunden Informationsasymmetrien vorliegen.

Auch innerhalb der Unternehmen gibt es eine „Rahmenordnung“ (Unternehmenskultur, Leitlinien etc.) für die in ihr handelnden Akteure. Zu ihr zählen alle inneren Strukturen, Kommunikations- und Entscheidungsprozesse im Unternehmen sowie unternehmensspezifische Werte beziehungsweise Leitbilder. Entsprechend formulierte Unternehmensregeln zwingen die Stakeholder des Unternehmens in Verbindung mit der Rahmenordnung auf der Makroebene gleichsam zu moralisch gewünschtem Verhalten (vgl. Göbel 2010). Aber auch im Unternehmen gibt es Lücken in den Rahmenbedingungen. Insbesondere die mangelnde Kontrolle des Unternehmens über seine Stakeholder und deren Verhalten ermöglicht den Stakeholdern - in erster Linie den Beschäftigten - Handlungen, die nicht im Sinne der unternehmerischen Zielsetzung sind und bisweilen sogar direkt zulasten des Unternehmens gehen. Dies gilt auch für das in ethischen Fragen gewünschte Verhalten: Die Rahmenordnung des Unternehmen und die unternehmerische Kontrolle reichen nicht aus, die Umsetzung moralischer Vorgaben zu gewährleisten. Selbst Win-Win-Situationen, in denen Unternehmen und Gesellschaft von einer Maßnahme profitieren, werden nicht umgesetzt, wenn der einzelne Mitarbeiter sich von der Umsetzung keine Vorteile verspricht (vgl. Suchanek 2007: 65-71).

Ursächlich für dieses Kontrollproblem sind wiederum asymmetrische Informationsverteilungen, in diesem Fall zwischen dem Unternehmen und seinem Agenten, dem 
Beschäftigten, hinsichtlich des Handelns des Agenten. Die Prinzipal-Agenten-Theorie beschreibt die aufkommenden Probleme bei asymmetrischer Informationsverteilung nach Vertragsabschluss zwischen zwei Vertragspartnern. Als Prinzipal gilt der Auftragsgeber, also zum Beispiel der Eigentümer einer Firma, der den Agenten, also den zuständigen Manager oder Mitarbeiter, zur Erfüllung einer Leistung bezahlt. Problem der Handlungsbeziehung ist, dass der Prinzipal den Agenten bei Ausführung der aufgetragenen Aufgabe nicht kontrollieren kann. Es liegen also asymmetrische Informationen vor, die dem Agenten einen diskretionären Handlungsspielraum bieten (vgl. Richter/Furubotn 2003: 224). Diese Freiheit im Handeln kann der Agent zu seinen Gunsten missbrauchen und so gegen den Willen des Prinzipals handeln. Die wirtschaftswissenschaftliche Literatur spricht hier von Moral Hazard (vgl. Voigt 2009: 102ff.). Moral Hazard betrifft den Konflikt nach Vertragsabschluss zwischen Prinzipal und Agenten. Der Prinzipal befindet sich in einer schwierigen Bewertungssituation, da die Leistung des Agenten nicht nur von seinen Handlungen und Entscheidungen, sondern auch durch vom Agenten nicht beeinflussbaren Faktoren determiniert wird. Ein hohes Maß an Komplexität der Arbeitsbedingungen macht es dem Prinzipal fast unmöglich, Erfolg oder Misserfolg genau wie ethisches oder unethisches Geschäftsgebaren direkt dem Verhalten und den Entscheidungen des Agenten zuzuschreiben.

Ein gebräuchliches Beispiel zur Verdeutlichung stellt eine Aktiengesellschaft dar, in der üblicherweise dem Prinzipal (Aktionär) weniger Informationen als dem Agenten (Vorstandsmitglied) vorliegen. Erwirtschaftet ein Unternehmen in einer Periode Verluste, wissen die Aktionäre nicht genau, ob hierfür exogene Schocks verantwortlich sind oder ob Managementversagen vorliegt. Moral Hazard ist gegeben, wenn der Manager sein falsch motiviertes Handeln mit den äußeren Einflussfaktoren begründet, obwohl er eigentlich selbst die Verantwortung für die eingehandelten Verluste trägt (vgl. Voigt 2009: 102f.). Eine Lösung des Prinzipal-Agenten-Problems wäre nun die Gestaltung eines Vertrages, der einerseits bestmöglich die Erwartungen des Prinzipals widerspiegelt und andererseits der Nutzenmaximierung des Agenten dient, damit die Handlungen des Agenten nach Vertragsabschluss genau in die vom Prinzipal intendierte Richtung weisen. Dabei sind allerdings die damit verbundenen Transaktionskosten zu berücksichtigen, denn es gilt permanent stattfindende Neuerungen und Änderungen der wirtschaftlichen Umgebung mit zu berücksichtigen. Genau wie aufgrund der asymmetrischen Informationsverteilung zwischen Regelsetzer und Regelnehmer staatlicher Gesetze ein vollständiges Rahmenwerk nicht formuliert werden kann, so kann auch auf Unternehmensebene kein perfekter Vertrag zur Steuerung des Agenten im Sinne des Prinzipals aufgesetzt werden. Die Prinzipal-Agenten-Theorie liefert eine exakte Beschreibung des Problems von Gesellschaften oder Organisationen, die Entscheidungen, Handlungen und Verhalten ihrer Agenten in die gesellschaftlich gewünschten Bahnen zu lenken. Dies betrifft sowohl die Ebene auf der die Gesellschaft als Prinzipal, dem Unternehmen als Agenten, den Auftrag zur Erfüllung der oben genannten Funktionen zur Förderung des Gemeinwohls erteilt, als die Unternehmen als Prinzipale bei der Steuerung ihrer Mitarbeiter.

Letztlich trägt daher auch jeder einzelne Wirtschaftsakteur, über die Regeln und Vorschriften der Gesellschaft und des Unternehmens hinaus, für seine Handlungen ent- 
sprechend der eigenen moralischen Maßstäbe Verantwortung. Zu den einzelnen Wirtschaftsakteuren zählen Kunden und Lieferanten (genauer: deren jeweilige Mitarbeiter), Arbeitnehmer und Investoren. Kennzeichnend für die Individualethik ist die Analyse des Einflusses von Personen, Unternehmen zu beeinflussen, zu verändern und weiterzuentwickeln. Das Unternehmen mag ein noch so konsistentes Leitbild und ethische Standards haben, ohne eine grundlegende Individualmoral werden diese kaum etwas bewirken können, da es immer Umgehungsmöglichkeiten gibt (vgl. Leisinger 1997: 141). Somit muss den Agenten, die die Unternehmensregeln umsetzen und leben sollen, besondere Bedeutung auch in der Unternehmensethik beigemessen werden denn sie haben den Informationsvorsprung.

\section{Konsumentenethik: Bedeutung und Grenzen}

Über die diskretionären Handlungsspielräume für Unternehmen ist sich die wirtschaftsethische Literatur weitgehend einig (vgl. Ulrich 1998; Homann/Lütge 2005). Um die Handlungen innerhalb dieser Spielräume in die gesellschaftlich gewünschten Bahnen zu lenken ist - so die Idee der Konsumentenethik - Druck von außerhalb, aus der Gesellschaft heraus, in das Unternehmen hinein erforderlich (vgl. Heidbrink et al. 2011: 28f.; Harrison et al. 2005). Für Unternehmen sind dabei die Konsumenten besonders wichtig, da sie durch den Kauf oder Nichtkauf der Unternehmensprodukte die wirtschaftliche Existenz des Unternehmens garantieren oder in Frage stellen. Denn handelt ein Unternehmen gegen die Erwartungen seiner Kunden, kann ihm durch Kaufboykott die Daseinsberechtigung am Markt, seine licence-to-operate, entzogen werden.

Somit können auch Konsumenten Träger ethischer Verantwortung sein, wenn und soweit sie bewusst Unternehmen, die gegen verbreitete Moral- und Wertvorstellungen handeln, durch den Boykott der Produkte sanktionieren. Kann sich ein Unternehmen durch sein vorbildliches, nachhaltiges Handeln eine positive Reputation im Auge der Konsumenten aufbauen, kann es hingegen dadurch seine Absatzsituation auf dem Markt verbessern (vgl. Brown/Dacin 1997: 68-84; Schrader 2003: 82). Da Konsumenten in einer Marktwirtschaft besonders machtvoll im Hinblick auf die Beeinflussung unternehmerischer Marktstrategien sind, müssen Unternehmen deren Interessen in den Mittelpunkt ihrer strategischen Planungen setzen: Ohne Nachfrager lässt sich kein Gewinn erzielen. Dadurch haben Konsumenten aus Sicht der Unternehmen mitunter auch die Verantwortung für die moralische Qualität der Produkte (vgl. Heidbrink et al. 2011: 32ff.). In dieser Perspektive wird der Kunde zum Prinzipal, der seinem Agenten, dem Unternehmen, direkt oder indirekt den Auftrag erteilt, ihm ein solches Produkt anzubieten, wie es seinen Bedürfnissen - auch seinen ethischen Wünschen - entspricht.

Die Bedeutung des Konsumenten in seinem Einfluss auf die Unternehmensentscheidungen wächst stetig seit dem Zweiten Weltkrieg. Gründe sind Wirtschaftswachstum und Fortschritt, die damit verbundene Abkehr von der Massenproduktion von Gütern hin zu individuellen Lösungen sowie ein steigendes Pro-Kopf-Einkommen (vgl. Wildt 1995; Korff/Feldhaus 1999). Der Wohlstand bringt es mit sich, dass mit einem Basisprodukt verbundene Differenzierungs- und Alleinstellungsmerkmale im Augenmerk 
der Konsumenten eine zentrale Rolle spielen. Wenn der Kunde konsumiert evaluiert er verschiedene Teilaspekte des Produktes, unter anderem können moralische Motive damit kaufentscheidend sein (vgl. Göbel 2010: 89).

Nachhaltige Produktionsbedingungen, die Umweltschäden verringern oder verhindern, werden in der Tat von einigen Konsumentengruppen vermehrt nachgefragt. Nach neuesten Studien interessiert sich die Mehrheit der Bürger für soziale und ökologische Standards; diese Präferenzen setzen jedoch nur rund zehn Prozent in Kaufentscheidungen um (vgl. Heidbrink et al. 2011: 9f.). Trotzdem kann seit einigen Jahren in Deutschland ein Wachstum beim Kauf von Bio-Produkten beobachtet werden. Die Verbindung von Nachhaltigkeit in Bezug auf die Natur sowie mit Blick auf die eigene Gesundheit funktioniert bei Bio-Produkten recht gut (vgl. Stehr 2007: 285ff.). Schwieriger ist dies hingegen bei fair gehandelten Produkten, denn dort steht den höheren Kosten „nur“ ein besseres Gewissen und nicht die bessere Gesundheit entgegen.

Dennoch wächst das Interesse der Konsumenten, Produkte zu unterstützen, die unter moralisch vertretbaren Arbeitsbedingungen hergestellt werden. Die Theorie des politischen Konsums bildet diese Tendenz auf den Märkten ab: Politischer Konsum beschreibt die Verfolgung von nachhaltigen wirtschaftlichen, sozialen und/oder ökologischen Zielen von Marktteilnehmern im öffentlichen Raum. Hierbei verschmelzen die zunächst unterschiedlichen Bedeutungen des politischen Bürgers und des ökonomischen Konsumenten zum Handeln eines aktiven Individuums, das durch seine Konsumfreiheit Kaufentscheidungen unter ethischen Gesichtspunkten trifft. Dabei lässt sich der politische Konsument entweder von Konfrontations-(Boykott) oder Kooperationsstrategien - dem oft so genannten „Buykott“ von „fairen“ Produkten - leiten (Neuner 2007: 342f.). Die intensive Nutzung westlicher Firmen von Arbeitskräften aus Niedriglohnländern - als ein Beispiel - wird mehr und mehr in der Öffentlichkeit kritisiert beziehungsweise abgelehnt, soweit die Arbeitsbedingungen dort als unwürdig empfunden werden. Kleidung zu tragen, die in ausbeuterischer Kinderarbeit oder von Frauen zu nicht existenzsichernden Löhnen und unter gesundheitsgefährdenden Arbeitsbedingungen hergestellt wurde, wird abgelehnt. Die Theorie des politischen Konsums würde hier dementsprechend einen Boykott vermuten. Gerade große Konzerne, die durch die Kombination von kostengünstiger Massenproduktion in Asien und hoher Preissetzung in ihren westlichen Absatzländern als Grenzmoralisten enorme Gewinne erwirtschaften konnten, hatten nach Bekanntwerden dieser Strategie mit Imageschäden zu kämpfen. Verschlechtert sich das Öffentlichkeitsbild und stößt das Produkt wegen ethischer Normverletzungen auf Ablehnung, nutzt der Kunde sein Drohpotenzial und sanktioniert die Firma durch Boykott (vgl. Heidbrink et al. 2011: 33; Harrison et al. 2005: 3). Es werden auch höhere Preise akzeptiert, wenn sie auf einem moralischen Mehrwert fundiert sind (vgl. Habisch 2003). Der Verbraucher kann so auf die Entwicklung von Produkten einwirken und aktiv Consumer Social Responsibility beziehungsweise politischen Konsum leben (vgl. Kendall et al. 2007; Delacote/MontagnéHuck 2011).

$\mathrm{Ob}$ und wieweit tatsächlich Rahmenbedingungen für nachhaltigen Konsum durch die Kaufentscheidung des Kunden beeinflusst werden, wird indes nach wie vor kontro- 
vers diskutiert. Die Bereitschaft, eine Präferenz aus ethischer Motivation zu entwickeln und dafür einen Preisaufschlag zu zahlen, ist von Kundentyp und Produktart abhängig. Zwischen 5 und 36 Prozent an Preisaufschlag gelten hierbei als vom Konsumenten akzeptiert (vgl. De Pelsmacker et al. 2005).

Die theoretische Kritik am Einfluss der Konsumentenethik basiert auf mehreren Argumenten:

- Ethische Maßstäbe sind nur ein Kaufgrund unter vielen: Der Großteil der Gesellschaft zieht überwiegend andere als moralische Bewertungsmaßstäbe beim Kauf heran. Der Kaufentscheidungsprozess erweist sich als schwer nachvollziehbar und komplex (vgl. Brinkmann 2004: 136). Ein günstiger Preis, das gute Ansehen der Marke oder andere vom Konsumenten als wichtig erachtete Differenzierungsmerkmale, aber auch die spontane Entscheidung am Verkaufsort, spielen eine große Rolle. Der Konsument handelt nur begrenzt rational: Er nutzt seinen Evoked Set oder lässt sich am Point of Sale beeinflussen (vgl. Reisch/Hagen 2011: 233).

- Der Status quo Bias verhindert die Umsetzung moralischer Präferenzen: Er beschreibt das Phänomen, eine Präferenz für den Ist-Zustand zu haben, obwohl dieser durchaus verbesserungswürdig und nicht dem Optimum entsprechend ist. Lieber wird an (unbegründeten) festgesetzten Regeln, Traditionen oder Kaufentscheidungen festgehalten, anstatt Neues zu wagen. So wird oftmals Fortschritt und Entwicklung gebremst. Zukünftiges wird grundsätzlich mit Unsicherheit assoziiert, während das Gewohnte für die Mehrheit das Gerechte repräsentiert (vgl. Enste et al. 2009). Ein Beispiel für die Folgen dieser Wahrnehmungsverzerrung zeigt sich im schleppenden Wechsel der Verbraucher zu ökologisch handelnden Stromanbietern. Obwohl das Bewusstsein zur Unterstützung der Nachhaltigkeit bei vielen vorhanden ist, bleiben sie ihrem alten, teurerem und umweltschädlicherem Anbieter treu.

- Als weiteres irrationales Verhaltensmuster beeinflusst derzeit das Herdenverhalten (Herd effect) die Umsetzung ethischer Präferenzen negativ: Die menschliche Kaufentscheidung wird durch den Drang zur Imitation gelenkt. Unterbewusst verleitet uns das Herdenverhalten dazu, diejenigen Produkte zu kaufen, die von uns als wichtig wahrgenommene Personen auch kaufen. Kommen die Leittiere der Herde ihrer ethischen Verantwortung im Konsum nicht nach, fällt es uns schwer, vom Herdenverhalten abzuweichen (vgl. Reisch/Hagen 2011: 223-230). Im Umkehrschluss gilt aber auch: Wenn sich die Herde erst verantwortungsbewusst verhält, ist dies ein zusätzlicher Druck für den Einzelnen, dies zu imitieren. In diese Richtung versuchen einige Schauspieler zu wirken, die für einen „Life Style of Health and Sustainability“ (LOHAS) werben. Aber bis ein Toyota Prius (als Hybridauto) ein ähnliches Statussymbol wird, wie die zahlreichen Geländewagen (SUV), wird wohl noch einige Zeit vergehen.

- Boykottdrohungen sind gerade bei marktmächtigen Unternehmen nur schwer möglich: Große und bekannte Marken werden zwar besonders häufig zum Ziel von Boykottkampagnen, weil diese mit ihrem Namen besonders viel Aufmerk- 
samkeit in der Öffentlichkeit erreichen und dadurch auch gleichzeitig die gesamte Protestaktion besondere Beachtung erhält (vgl. Palazzo/Basu 2007). Das Phänomen des Boykotts bekannter Marken kann schon seit Jahrzehnten in „Anti-Sweat-Shop“-Aktionen politischer Konsumenten verfolgt werden. Es ist ein eindeutig wachsendes Bemühen der Bevölkerung zu erkennen, konkreten Einfluss auf Unternehmensprozesse auszuüben (vgl. Stolle et al. 2005: 247f.). Besteht jedoch - und dies ist bei bekannten Marken meist der Fall - eine Situation der Marktmacht, wird ein Boykott dieser Marke oft mit einem Komplettverzicht auf die entsprechenden Produkte einhergehen, weil keine adäquaten Substitute der Konkurrenz vorhanden sind. Ist das Produkt dem Konsumenten wichtig, wird er daher seine moralischen Präferenzen zurückstellen und die Marke weiter kaufen.

- Uninformierte Kunden kennen oft die ethischen Standards der Unternehmen nicht: Trotz der Tatsache, dass der Konsument im Zeitablauf quantitativ und qualitativ an Bedeutung im Marktprozess gewonnen hat und die moderne Gesellschaft des Westens von manchem Autor daher als consumer society bezeichnet wird (vgl. Keat et al. 1994: 3), herrscht keineswegs vollständige Konsumentensouveränität. Als oberstes Ziel von Unternehmen gilt nach neoklassischer ökonomischer Theorie zwar die Bedürfnisbefriedigung der Kunden (vgl. Knobloch 1994: 77ff.). Es kann jedoch nicht davon gesprochen werden, dass sich das Angebot perfekt an die Bedürfnisse der Nachfrage anpasst. Die potenziellen Kunden sind in ihrer Informationsbeschaffung gegenüber Produkt- und Unternehmenscharakteristiken von der Auskunftsbereitschaft der jeweiligen Firma abhängig. Da die Sozial- oder Umweltverträglichkeit eines Produktionsprozesses vom Kunden nicht durch bloßes Betrachten festgestellt werden kann, fallen oftmals nicht nur hohe Informationssuchkosten für den Nachfrager an bei Informationsasymmetrie lässt dieses Defizit sich nachfrageseitig auch nicht überwinden.

- Für die Unternehmen besteht damit die Möglichkeit zur Manipulation der Kunden: Der ethisch motivierte Kunde, der als verantwortungsbewusster Prinzipal dem Unternehmen den Auftrag gibt, ein nachhaltiges Gut (zum Beispiel ein Fair-Trade-Produkt) zu produzieren, stößt in seinen Kontrollmöglichkeiten schnell an Grenzen. Ungeschützte Begriffe, schwammige Werbebotschaften oder fehlendes Expertenwissen erschweren es ihm, seine moralische Präferenz in die Tat umzusetzen. Auch hier wirken somit die Folgen asymmetrischer Informationen: Der Kunde kann nicht kontrollieren, ob die Firma wirklich seinen Erwartungen entsprechend gehandelt hat oder doch profitmaximierende Leitsätze dominiert haben (vgl. Kaas 1992). Er ist somit gar nicht in der Lage, selbst wenn er wollte, vollständig fairem Konsum nachzugehen, der ein wirtschaftliches wie auch soziales Gleichgewicht herstellt, da ihm die benötigten Informationen zur Bewertung fehlen (vgl. Neuner 2007: 343). Stellt er dennoch fest, dass das Unternehmen sich nicht wie gewünscht verhält, kann er allenfalls mittels Boykottdrohung versuchen, eine Strategieänderung anzustoßen. Inwieweit ein Nachfragerückgang das Unternehmen aber tatsächlich dazu bewegt, 
unmoralisches Verhalten zu ändern, hängt von verschiedenen Determinanten ab. Insbesondere die Reichweite des genutzten Drohpotenzials müsste sehr groß sein und die Kosten der Drohung eher gering, um Umsatzeinbrüche bei einem Global Player zu bewirken. Zwar wird nach öffentlichen Skandalen aufgrund von Arbeitsrechtverletzungen oder Umweltverschmutzungen oft ein neues, nachhaltiges Konzept vorgelegt. Inwieweit dieses danach aber realisiert wird, bleibt fraglich. Es reicht aus Unternehmenssicht ja bei Informationsasymmetrie aus, den Kunden glauben zu lassen, die Strategie habe sich geändert.

- Die Kunden wissen wiederum um die Manipulationsmöglichkeit und können daher systematisch ethische Standards bei der Kaufentscheidung ignorieren: Betreibt ein Unternehmen gesellschaftliches Engagement, muss es dies kommunizieren, um die Informationsasymmetrie zu überwinden und eine Reaktion seiner Kunden zu erhalten. Wirbt eine Firma zu penetrant mit ihrem außerordentlichen Bemühen oder führt bloß eine wahllose Aneinanderreihung oder Aufzählung von CSR-Maßnahmen an, kann diese entsprechende Werbung der Unternehmen aufgrund des aufkommenden Manipulationsverdachtes kontraproduktiv wirken. Jede freiwillige soziale Tätigkeit muss glaubwürdig von der Organisation beworben werden und nicht in Verdacht geraten, Teil des sogenannten Greenwashing zu sein. Als Faktoren, welche die Glaubwürdigkeit eines Unternehmens unterstützen, gelten zum einen die Verbindung von Kerngeschäft und Übernahme von gesellschaftlicher Verantwortung in ergänzenden Bereichen sowie eine Kalkulation, in der die Kosten für die Kommunikation nicht die Kosten für das eigentliche Engagement überschreiten (vgl. Yoon et al. 2006).

Da der Konsument aus seiner Position als Prinzipal am Ende der Wertschöpfungskette heraus meist nicht in der Lage ist, genügend Informationen über die „Moralität“ eines Produktes zu sammeln, wird er von staatlicher und gesellschaftlicher Seite unterstützt. Die Einführung von Normen, Standards oder Symbolen soll Transparenz schaffen. Die Unternehmen verpflichten sich, nach denjenigen Vorschriften zu wirtschaften, zu denen sie sich bekannt haben. Ist der Kunde tatsächlich bereit, mehr für ein nachhaltiges Produkt zu zahlen, profitiert auch das partizipierende Unternehmen davon, da sein Gut gegenüber dem der Konkurrenz hervorsticht. Die Informationsasymmetrie wird durch Normen und Standards behoben, das Nichteinnehmen der Position des Grenzmoralisten kann honoriert werden. Noch herrscht auf dem Markt jedoch eine hohe Intransparenz durch eine zu große Zahl an Richtlinien und Siegeln, die zum Teil eher verwirrend anstatt klärend empfunden werden. Bis zu einer weitreichenden Konsumentensouveränität auch in ethischen Fragen scheint es noch ein weiter Weg zu sein.

Ein weiterer Grund für die bisher begrenzt ausgeübte Macht der Konsumenten kann in ihrem vergleichsweise geringen Involvement, das heißt der Ich-Beteiligung und der Bedeutung einer Entscheidung für die eigene Person, liegen (vgl. KroeberRiel/Weinberg 2003). Damit ist nicht gemeint, dass Konsum nicht wichtig ist - man denke nur an die Bedeutung von Statussymbolen -, sondern dass die Rolle als Konsument im Hinblick auf moralische Standards weniger bedeutsam ist, als die Rolle als 
Mitarbeiter in einem Unternehmen, mit dem man sich sehr viel stärker identifiziert, als mit den Produkten, die gekauft werden. Deren Rolle beleuchtet der folgende Abschnitt zur Agentenethik.

\section{Agentenethik: Implikationen und empirische Befunde}

Besser informiert als der Kunde als Prinzipal des unternehmerischen Handelns ist der Mitarbeiter als Agent des Unternehmens. Er kann im Produktionsprozess unmittelbar beobachten, inwieweit ökologische Aspekte beachtet und soziale Standards ernst genommen werden. Die Mitarbeiter des Unternehmens sind folglich als Agenten besser informiert als die Prinzipale, seien es Eigentümer, Aktionäre oder Kunden. Dafür kann der Agent allerdings nicht mit einem Kaufboykott drohen. Wie also soll er den gelegentlich auftretenden Zielkonflikt zwischen Unternehmenszielen, individuellem Agentennutzen und Gewissen respektive Gemeinwohlorientierung lösen?

Wo Individualethik einen Zielkonflikt konstituiert, ist bekanntlich die Überführung in eine orthogonale Positionierung gefragt (vgl. Pies/Sardison 2005; Pies 2008; Pies 2011). Pies hat dies in seinem ordonomischen Forschungsprojekt wie folgt herausgearbeitet: Zur Überführung des sozialen Dilemmas, welches sich dem Agenten stellt, dient das Drei-Ebenen-Schema. In der ordonomischen Perspektive lässt sich der Zielkonflikt im Basisspiel innerhalb des Metaspiels oder des Meta-Metaspiels auflösen (vgl. Pies et al. 2009). Im Basisspiel geht es um die Regelbefolgung respektive die Spielzüge in einer gegeben Rahmenordnung. Im Metaspiel geht es um die Festsetzung der Spielregeln respektive eines institutionellen Rahmens für die Interaktionen. Im Metaspiel werden Regeln vereinbart, in denen das Basisspiel stattfindet. Das MetaMetaspiel hingegen widmet sich dem Diskurs über die Regelsetzung. Das Metaspiel betrifft den Regelsetzungsprozess, das Meta-Metaspiel den Regelfindungsdiskurs. Dabei haben die Agenten verschiedene Möglichkeiten:

Erstens: Sind die Agenten im Unternehmen in einer Position, in der sie über die Einhaltung der ethischen Standards selbst mitbestimmen können, so haben sie eine Stimmoption - sie können mitentscheiden, ob moralische Standards Beachtung finden oder nicht. Dann können sie nicht nur im Meta-Metaspiel, sondern auch im Metaspiel agieren.

Zweitens: Haben sie eine sich aus der Möglichkeit, das Unternehmen zu verlassen, ergebende Drohoption. Diese Drohoption war auf dem deutschen Arbeitsmarkt über viele Jahre für einen großen Teil der Beschäftigten keine Option mit tatsächlichem Drohpotenzial. Der deutsche Arbeitsmarkt zeigte, in den 90er Jahren bis hinein ins letzte Jahrzehnt, eine hohe Arbeitslosigkeit. Viele Arbeitskräfte waren daher froh, überhaupt eine Arbeitsstelle innezuhaben. Dies ist jedoch im Änderungsprozess begriffen, die Arbeitslosenquoten sind gesunken und in vielen Qualifikationsfeldern kommt ein Mangel an Fachkräften auf die Unternehmen zu. Damit hat sich die Verhandlungssituation von Spezialisten gebessert: Auf dem Markt haben Fachkräfte ein wirksames Drohpotenzial durch ihre Möglichkeit zur Konkurrenz zu wechseln, wenn von ihnen gewünschte ethische Standards nicht eingehalten werden. Zwar haben sol- 
che Fachkräfte im Metaspiel keine Spielberechtigung, dafür aber im Meta-Metaspiel einen erheblichen Einfluss auf den unternehmensinternen Diskurs.

Wer als Mitarbeiter über Stimm- und über Drohoption verfügt, ist somit ein relevanter Agent ethischen unternehmerischen Verhaltens. Hält er moralische Standards hoch, so sind die ihn umgebenden Entscheidungsträger angehalten und die ihm unterstellten Mitarbeiter gezwungen, ebenfalls moralische Standards zu beachten, soweit der relevante Agent hierüber informiert ist. Dies gilt für Konsumenten, Unternehmenseigner, Kollegen aber auch Mitarbeiter, die weder über eine Stimm- noch über eine Drohoption verfügen.

Drittens: Verfügen die Mitarbeiter aber über die Resignationsoption: Denn wer auf der informierten Seite ist - also mithin einen entscheidungsirrelevanten Agenten darstellt - kann zumindest innerlich abwandern, wenn die Nichteinhaltung ethischer Standards seinen Bedürfnissen und Ansichten widerspricht. Ein informierter Mitarbeiter ohne Entscheidungskompetenz kann innerlich kündigen, seine Produktivität und sein Engagement zurückfahren und Dienst nach Vorschrift leisten. Hier lässt sich von einer Resignationsoption sprechen. Für das Unternehmen wie auch für die Gesellschaft ist es zentral, dass entscheidungsrelevante wie irrelevante Agenten motiviert ethischen Standards nachkommen, um ein produktives Zusammenleben zu gewährleisten - sie sollen keinesfalls die Resignationsoption ziehen. Für das Unternehmen wirkt diese Option jedoch ebenfalls als Beschränkung unethischen Verhaltens.

Die drei Optionen verdeutlichen, wie wichtig der Aufbau einer Unternehmenskultur im gemeinsamen Diskurs ist, die klare Werthaltungen transportiert und diese konkret auch als Verhaltensmaximen an die Beschäftigten weitergibt (vgl. Kuhlen 2005: 38). Werte wie Ehrlichkeit, Leistungsbereitschaft oder Eigenverantwortlichkeit der Agenten können durch die Implementierung einer konsistenten Unternehmenskultur gefördert werden, da sie dann als Orientierungsmaßstab herangezogen werden und dadurch direkt auf soziale Austauschprozesse einwirken (vgl. Beckmann/Pies 2006). Für eine solche Unternehmenskultur sind primär nicht die Shareholder oder die Konsumenten, sondern die Agenten selbst verantwortlich: Sie agieren miteinander und sie verfügen über einen Informationsvorsprung.

Als besondere Ausprägungen des ethisch motivierten Handelns von Unternehmen, respektive ihrer Agenten, gelten Corporate Social Responsibility und Corporate Citizenship. Wo Corporate Social Responsibility über die Unternehmenskernfunktionen hinaus Mitverantwortung für die Gesellschaft von den Unternehmen einfordert, beinhaltet Corporate Citizenship die Teilhabe der Unternehmung an gesellschaftlichen Lenkungsprozessen (vgl. Beschorner 2008). Während Corporate Social Responsibility auch die unbeobachtbaren, in der Struktur und Kultur des Unternehmens implementierten Normen und Werte beinhaltet, konzentriert sich Corporate Citizenship eher auf die beobachtbare Teilnahme am gesellschaftlichen Diskurs, mithin um das Mitwirken in der Gesellschaft am Metaspiel und am Meta-Metaspiel. Hier kann der Konsument noch vergleichbar gut beobachten, ob und inwieweit ein Unternehmen seiner gesellschaftlichen Mitverantwortung nachkommt. Dass aber sogar im bürgerschaftlichen Engagement der Unternehmen, wo die Wirkungen für die Öffentlichkeit und 
damit auch für den Konsumenten im Vergleich zur Beachtung von Moral und gesellschaftlicher Verantwortung in den Produktionsbedingungen gut sichtbar werden, der Agent mit seiner Motivation eine bedeutsame Rolle spielt, demonstrieren die Ergebnisse des Ersten Engagementberichts 2012 der Bundesregierung.

Eine deutliche Mehrheit der befragten Unternehmen bestätigen im Rahmen des Ersten Engagementberichts die zentrale Relevanz der Unternehmenskultur: 76,4 Prozent der Unternehmen geben an, ihr bürgerschaftliches Engagement sei Teil ihrer Unternehmenskultur. Besonders Großunternehmen mit über 500 Mitarbeitern bestätigen diesen Punkt: Von ihnen engagieren sich über 90 Prozent im Rahmen der Unternehmenskultur. In der Zukunft wird die Implementierung des bürgerschaftlichen Engagements im Rahmen von Unternehmenskultur und Agentenethik noch weiter ansteigen: Fast die Hälfte derjenigen Unternehmen, die ihr bürgerschaftliches Engagement im Rahmen ihrer Unternehmenskultur betreiben, gehen davon aus, dass die Bedeutung von bürgerschaftlichem Engagement in den nächsten fünf Jahren zunehmen wird (siehe Tabelle 1). Dem gegenüber äußern nur 29 Prozent der Unternehmen, die bürgerschaftliches Engagement ohne die Einbettung in ihre Unternehmenskultur betreiben, dass bürgerschaftliches Engagement an Bedeutung zunehmen wird. Man kann dies in zwei Wendungen deuten: Den Agenten in den Unternehmen, die sich im Rahmen einer Unternehmenskultur ethisch verantwortlich zeigen, erschließt sich die zunehmende Bedeutung dessen auch für die Gesellschaft. Oder andersherum: Wer an eine zunehmende Bedeutung des bürgerschaftlichen Engagements glaubt, ist eher bereit, dieses in eine einschlägige Unternehmenskultur einzubetten.

\begin{tabular}{|c|c|c|c|c|}
\hline \multirow{2}{*}{\multicolumn{2}{|c|}{$\begin{array}{l}\text { Befragte Unternehmen, die sich bürger- } \\
\text { schaftlich engagieren }\end{array}$}} & \multicolumn{3}{|c|}{$\begin{array}{l}\text { Erwarten Sie, dass die Bedeutung von } \\
\text { bürgerschaftlichem Engagement in Ihrem } \\
\text { Unternehmen in den nächsten } 5 \text { Jahren... }\end{array}$} \\
\hline & & ...zunimmt & $\begin{array}{l}\text {...gleich bleibt } \\
\text { oder abnimmt }\end{array}$ & Alle \\
\hline \multirow[b]{2}{*}{$\begin{array}{l}\text { Bürgerschaftliches } \\
\text { Engagement }\end{array}$} & $\begin{array}{l}\text {...im Rahmen der } \\
\text { Unternehmenskultur } \\
\text { als Element der } \\
\text { Agentenethik }\end{array}$ & $46 \%$ & $54 \%$ & $100 \%$ \\
\hline & $\begin{array}{l}\text {...ohne Einbettung in } \\
\text { die Unternehmens- } \\
\text { kultur }\end{array}$ & $29 \%$ & $71 \%$ & $100 \%$ \\
\hline
\end{tabular}

Tabelle 1: Agentenethik als Baustein für die zukünftige Weiterentwicklung des bürgerschaftlichen Engagements der Unternehmen; N=1.992 Unternehmen in Deutschland (Quelle: Sonderauswertung der Daten der Unternehmensbefragung für den Ersten Engagementbericht 2012) 
Zusätzlich zeigt sich auch die Mitarbeitermotivation als von hoher Bedeutung für die Unternehmen, soziales Engagement zu betreiben: 44,3 Prozent der Unternehmen nennen sie als ein Ziel. Zusammen mit der Nennung von Qualifizierung und Entwicklung der sozialen Kompetenz der Mitarbeiter von 42,1 Prozent der engagierten Unternehmen als weiterem Ziel, spiegelt dies das besondere Interesse der Unternehmen wider, seine Beschäftigten, die Agenten, in den Fokus ethischer Überlegungen miteinzubeziehen. Bürgerschaftliches Engagement soll vor diesem Hintergrund dem Nutzen der Gesellschaft, mithin in der Perspektive der Prinzipal-Agenten-Theorie sowohl dem der Agenten als auch dem der Prinzipale, dienen.

Allerdings wird im Engagementbericht auch der im Vergleich für die Unternehmen ebenfalls hohe Stellenwert der Konsumenten deutlich: 46,4 Prozent der befragten Unternehmen geben die Erhöhung der Kundenbindung beziehungsweise die Gewinnung neuer Kunden als einen Nutzen bürgerschaftlichen Engagements an. Lediglich die Shareholder, die vor allem auf die Entwicklung des Unternehmenswertes achten, sind eher selten ein Ziel bürgerschaftlichen Engagements: Nur 33,1 Prozent der Unternehmen unterstützen durch bürgerschaftliches Engagement die Entwicklung des Markenwertes (vgl. Erster Engagementbericht der Bundesregierung 2012: 646).

Im Rahmen der Befragung des Ersten Engagementberichts wurde nicht nur eruiert, für welche Anspruchsgruppe das Engagement primär gedacht ist, sondern auch gefragt, inwieweit das Unternehmen der Auffassung ist, dass durch die Befriedigung der Wünsche der jeweiligen Stakeholdergruppe dem Unternehmen ein Nutzen entstehe, mithin ob eine Win-Win-Situation generiert werde. Abbildung 1 illustriert in Balkenform diese Zielerreichung: Diejenigen, die mit ihrem gesellschaftlichen Engagement das Ziel der Mitarbeitermotivation anstrebten, erreichten es zu einem Anteil von 73,5 Prozent komplett oder zumindest teilweise (vgl. Erster Engagementbericht 2012: 856). Auch in der Mitarbeiterqualifikation und in der Vernetzung von Experten - beides ist ebenfalls ein Entgegenkommen an die Interessen der Agenten des Unternehmens - ist bürgerschaftliches Engagement nach Auskunft der Unternehmen ähnlich erfolgreich wie in der Kundenbindung und -gewinnung. Die Abbildung zeigt, dass auf Konsumenten wie Agenten nach Auffassung der Unternehmen mit ihrem bürgerschaftlichen Engagement ein ähnlich hoher Einfluss genommen werden kann. 


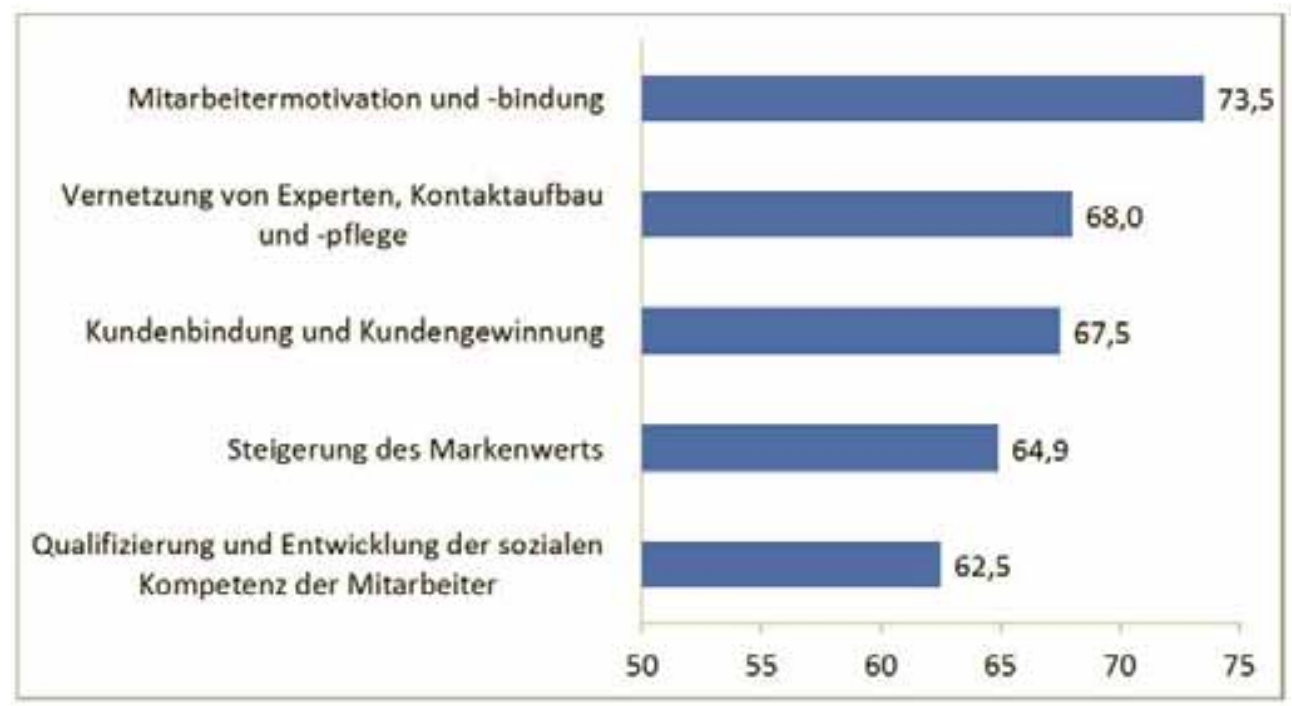

Abbildung 1: Welche Ziele können die deutschen Unternehmen mit gesellschaftlichem

Engagement ganz oder teilweise erreichen? Angaben in Prozent; $N=2.316$

Unternehmen in Deutschland (Quelle: Erster Engagementbericht 2012)

Hintergrundinformation: 2012 erscheint der Erste Engagementbericht der Bundesregierung mit einem Schwerpunkt auf dem Engagement der Unternehmen in Deutschland. Bürgerschaftliches Engagement wird hier als freiwilliges Mitwirken von Individuen und Organisationen im öffentlichen Raum definiert, welches strukturbildend und impulsgebend für das gesellschaftliche Miteinander sein soll. Dabei wird primär kein finanzieller, sondern ein sozialer Nutzen angestrebt. Der Erste Engagementbericht informiert über die aktuelle Lage Deutschlands und gibt unter anderem Auskunft über Motive, Kosten und Ziele von bürgerschaftlichem Engagement der Unternehmen. Datengrundlage ist dabei eine repräsentative Unternehmensbefragung im Rahmen des Zukunftspanels der IW Consult, in dem 4.392 Firmen zu verschiedensten Aspekten bezüglich ihrer freiwilligen Verantwortungsübernahme zu gesellschaftlichen Zwecken befragt wurden (vgl. Erster Engagementbericht 2012: 6).

Hierbei wurde eine Mehrheit von knapp zwei Drittel der Unternehmen identifiziert, die bürgerschaftliches Engagement betreiben (vgl. Erster Engagementbericht 2012: 7). Dabei steigt die Engagementquote mit zunehmender Unternehmensgröße und Mitarbeiterzahl; Großunternehmen engagieren sich sogar zu 96 Prozent für die Gesellschaft. Besonders intensiv werden in diesem Zusammenhang die Bereiche Soziales und Integration, Sport und Freizeit sowie Erziehung, Kindergarten und Schule durch hauptsächlich finanzielle Unterstützung gefördert.

Die Umfragedaten geben zudem Hinweise zur Frage der Zuständigkeit der ethischen Verantwortung: Denn die Unternehmen reagieren in ihrem Engagement darauf, welche Anspruchsgruppen ein Engagement von ihnen wünschen oder fordern. Im Rahmen der Befragung wurde unter anderem eruiert, welche Zielgruppe mit dem Enga- 
gement der Unternehmen angesprochen wird und inwieweit diese Ansprache von den Unternehmen als erfolgreich evaluiert wird. Einige Daten werden hier exklusiv vorgestellt.

\section{Fazit und Handlungsempfehlungen}

Unternehmen sind Institutionen, die - idealerweise in Form von Win-Win-Situationen - sowohl ihrem eigenen als auch dem Gemeinwohl dienen sollen. Doch oftmals behindern die bestehenden Informationsasymmetrien zwischen Unternehmensinterna und der Wahrnehmung des unternehmerischen Handelns in der Öffentlichkeit Marktprozesse. So wird es staatlichen Institutionen erschwert, eine Rahmenordnung, welche die Unternehmen im Basisspiel durchgehend zu moralischem Verhalten bewegt, durchzusetzen. Daher ist im Meta-Metaspiel ihre Diskursverantwortung gefragt. Betrachtet man die Informationsasymmetrien durch die Brille der Prinzipal-AgentenTheorie, so muss und möchte der Agent - und dies ist vor allem der Mitarbeiter des Unternehmens, der über Stimm- oder zumindest Drohoption verfügt - die Asymmetrie überwinden. Er will, dass sich sein Unternehmen am gesellschaftlichen Diskurs beteiligt und zugleich möchte er in den unternehmensinternen Diskurs involviert sein, in dem die Leitlinien und die Kultur des Unternehmens entwickelt werden.

Wo der Konsument nur unzureichend informierter Prinzipal, mit der begrenzten Sanktionsmöglichkeit der Boykottoption, ist, kann der Mitarbeiter mit seiner Stimmoption, seiner Drohoption oder mit seiner Resignationsoption erheblich mehr Einfluss geltend machen als der Konsument und ist zugleich besser über die Unternehmensinterna informiert und stärker involviert. Es verwundert nicht, dass die Befragung im Rahmen des Ersten Engagementberichts sogar beim vergleichsweise gut zu beobachtenden gesellschaftlichen Engagement der Unternehmen zum Ergebnis kommt, dass die Motivation und Qualifikation der Mitarbeiter ähnlich erfolgreich zu verfolgende Ziele moralischen Handelns der Unternehmen darstellen wie die Kundengewinnung und -bindung.

Unternehmenskultur und Mitarbeitermotivation lassen sich koppeln, da die Einbindung sozialer Elemente in die Unternehmenskultur, die die Kommunikation unter den Mitarbeitern fördern, zum einen Arbeitsprozesse erleichtern und zum anderen produktivere und motivierte Arbeitnehmer zur Folge haben (vgl. Putnam 1995). Dadurch wird die Wahrscheinlichkeit zur Realisierung der vom Agenten erwünschten Verhaltensweisen klar erhöht. Des Weiteren hilft dieses Verhalten Unternehmen, die Nachhaltigkeit als Teil ihrer Langzeitstrategie verankern, umweltfreundlich produzieren oder sich allgemein eine Reputation als ethisch engagierte Organisation aufbauen, leistungsmotivierte und ehrlichere Mitarbeiter anzuwerben. Auch das unerwünschte Ausnutzen von diskretionären Handlungsspielräumen gegenüber den Prinzipalen, also „Shirking“ am Arbeitsplatz, ist in Firmen mit einschlägiger Unternehmenskultur weniger verbreitet (vgl. Brekke/Nyborg 2008). Im Gegenteil zeigen Personen, die sich auf Grund des positiven, sozialen Rufs bei einer Firma bewerben, oftmals schon vorher in ihrem Privatleben ethische Bemühungen, die über die des Durchschnittsbürgers hinausgehen. Durch frühzeitiges Erkennen eigenständiger Initiativen der Mitarbeiter etwa in wohltätigen Verbänden oder Kirchen können schon im Rekrutierungsprozess die- 
jenigen Bewerber selektiert werden, die dann auch zukünftig eher die moralischen Bemühungen der Organisation unterstützen werden (vgl. Wang et al. 2010). Zusätzlich werden dann im Betrieb durch weitere Konzentration auf soziale Aspekte die Motivation, Loyalität und das Verantwortungsbewusstsein der Mitarbeiter gestärkt (vgl. Schaller/Bachmann 2004).

Die Agenten sollten sich intensiver mit dem Thema Ethik am Arbeitsplatz auseinandersetzen, um ihrer Diskursverantwortung gerecht werden zu können. 90 Prozent der im Rahmen des Ersten Engagementberichts befragten Unternehmen stimmen der Aussage zu, dass „Wirtschafts- und Unternehmensethik ein Pflichtfach in der wirtschaftswissenschaftlichen Ausbildung an Hochschulen sein sollte" (Erster Engagementbericht 2012: 882). In diesen Kursen lernen angehende unternehmerische Entscheidungsträger, ihre Entscheidungen nach ökonomischen und ethischen Gesichtspunkten abzuwägen (vgl. Kliemt 2008: 81ff.), um somit auf den nicht seltenen Konfliktfall in der Firma vorbereitet zu sein. Besitzt ein Entscheidungsträger durch ein solches Fach eine Basisbildung, steigt die Wahrscheinlichkeit, ethisches Verhalten auch im Berufsleben anzuwenden und der gesellschaftlichen Mitverantwortung adäquat nachzukommen. Oftmals werden dabei schon Präferenzen zu langfristig effizienten und moralischen Handlungen gelegt, die das verbreitete, kurzzeitige Streben nach Profit bremsen. Der Herdentrieb des Agenten, sich vom Druck der Konkurrenz verleiten zu lassen und die moralische Qualität der Handlungen zu ignorieren, kann so auf der Individualebene ins Gegenteil gedreht werden.

\section{Literaturverzeichnis}

Beckmann, M./Pies, I. (2006): Ordnungsverantwortung -Konzeptionelle Überlegungen zugunsten einer semantischen Innovation, Halle. Martin-Luther-Universität Halle-Wittenberg.

Beschorner, T. (2008): Corporate Social Responsibility und Corporate Citizenship: Theoretische Perspektiven für eine aktive Rolle von Unternehmen, in: Backhaus-Maul, H./Biedermann, C./Nährlich, S./Polterauer, J. (Hrsg.) (2010): Corporate Citizenship in Deutschland - Gesellschaftliches Engagement von Unternehmen. Bilanz und Perspektiven, 2. Auflage, Wiesbaden: VS Verlag, 111-130.

Brekke, K. A./Nyborg, K. (2008): Attracting Responsible Employees: Green Production as Labor Screening, in: Resource and Energy Economics Vol. 30/No. 4, 509-526.

Brinkmann, J. (2004): Looking at Consumer Behaviour in a Moral Perspective, in: Journal of Business Ethics, Vol. 51/No. 2,129-141.

Brown, T./Dacin, P. (1997): The Company and the Product: Corporate Associations and Consumer Product Responses, in: Journal of Marketing, Vol. 61/No. 1 68-84.

Delacote, P./Montagné-Huck, C. (2011): Political consumerism and public policy: Good complements against market failures, in: Ecological Economics, Vol. 73/Issue C, 188-193.

De Pelsmacker, P./Driesen, L./Rayp, G. (2005): Do Consumers Care About Ethics? Willingness to Pay for Fair Trade Coffee, in: Journal of Consumer Affairs (2006): Vol. 39/No. 2, 363-385, Winter.

Enste, D./Haferkamp, A./Fetchenbauer, D. (2009): Unterschiede im Denken zwischen Ökonomen und Laien. Erklärungsansätze zur Verbesserung der wirtschaftspolitischen Beratung, in: Perspektiven der Wirtschaftspolitik, Vol. 10,/No. 1, 60-78. 
Erster Engagementbericht der Bundesregierung (2012): Für eine Kultur der Mitverantwortung. Bericht der Sachverständigenkommission an das Bundesministerium für Familie, Senioren, Frauen und Jugend, Köln; Berlin.

Göbel, E. (2010): Unternehmensethik, 2. Aufl., Stuttgart: Lucius \& Lucius.

Habisch, A. (2003): Corporate Citizenship - Gesellschaftliches Engagement von Unternehmen in Deutschland, Berlin; Heidelberg; New York: Springer.

Harrison, R./Newholm, T./Shaw, D. (2005): Introduction, in: Harrison, R./Newholm, T./Shaw, D. (Ed.): The Ethical Consumer, London: Sage.

Heidbrink, L./Schmidt, I. (2011): Das Prinzip der Konsumentenverantwortung - Grundlagen, Bedingungen und Umsetzungen verantwortlichen Konsums, in: Heidbrink, L./Schmidt, I./Ahaus, B. (Hrsg.): Die Verantwortung des Konsumenten, Frankfurt a.M.: Campus, 25-56.

Heidbrink, L./Schmidt, I./Ahaus, B. (2011): Einleitung - Der Konsument zwischen Markt und Moral, in: Heidbrink, L./Schmidt, I./Ahaus, B. (Hrsg.): Die Verantwortung des Konsumenten, Frankfurt a.M.: Campus, 9-22.

Homann, K./Blome-Drees, F. (1992): Wirtschafts- und Unternehmensethik, Göttingen: UTB.

Homann, K./Lütge, C. (2005): Einführung in die Wirtschaftsethik, 2. Auflage, Münster: Lit Verlag.

Kaas, K. P. (1992): Marketing für umweltfreundliche Produkte, in: Die Betriebswirtschaft, Jg. 52/Heft 4, 473-487.

Keat, R./Whiteley, N./Abercrombie, N. (1994): Introduction, in: Keat, R./Whitley, N./Abercrombie, N. (Ed.): The Authority of the Consumer, London: Routledge, 1-19.

Kendall, B. E./Gill, R./Cheney, G. (2007): Consumer Activism and Corporate Social Responsibility - How Strong a Connection?, in: May, S./Cheney, G./Roper, J. (Ed.): The Debate over Corporate Social Responsibility, Oxford: University Press, 241-264.

Kliemt, H. (2008): Wirtschaftsethik als Lehrfach, in: Schwalbach, J. (Hrsg.): Corporate Social Responsibility (ZfB-Special Issue 3/2008), Wiesbaden: Gabler, 81-89.

Knobloch, U. (1994): Theorie und Ethik des Konsums, Bern: Paul Haupt.

Korff, W./Feldhaus, S. (1999): Handbuch der Wirtschaftsethik, Band 3, Gütersloh: Gütersloher Verlagshaus.

Kroeber-Riel, W./Weinberg, P. (2003): Konsumentenverhalten, 8. Aufl., München: Vahlen.

Kublen, B. (2005): Corporate Social Responsibility (CSR), Die ethische Verantwortung von Unternehmen für Ökologie, Ökonomie und Soziales, Baden-Baden: Deutscher Wissenschafts-Verlag.

Leisinger, K. M. (1997): Unternehmensethik - Globale Verantwortung und modernes Management, München: C. H. Beck.

Neuner, M. (2007): Politischer Konsum - Wesen, Erscheinungsformen und Bedeutung, in: WiSt- Wirtschaftswissenschaftliches Studium, Jg. 36/Nr. 7, München; Frankfurt a.M.: 342-347.

Noll, B. (2002): Wirtschafts- und Unternehmensethik in der Marktwirtschaft, Stuttgart; Berlin; Köln: Kohlhammer.

Palazzo, G./Basu, K. (2007): The Ethical Backlash of Corporate Branding, in: Journal of Business Ethics, Vol. 73/No. 4, 333-346.

Pies, I. (2008): Markt und Organisation. Programmatische Überlegungen zur Wirtschafts- und Unternehmensethik, Halle: Universität Halle-Wittenberg. 
Pies, I. (2011): Walter Eucken als Klassiker der Ordnungsethik - Eine ordonomische Rekonstruktion, in: Zeitschrift für Wirtschafts- und Unternehmensethik (zfwu), Jg. 12/Nr. 2, 222-249.

Pies, I./Hielscher, S./Beckmann, M. (2009): Moral Commitments and the Societal Role of Business. In: Business Ethics Quarterly, Vol. 19/No. 3, 375-401.

Pies, I./Sardison, M. (2005): Wirtschaftsethik, Lutherstadt Wittenberg: Wittenberg Zentrum für Globale Ethik.

Putnam, R. (1995): Bowling Alone: America's Declining of Social Capital, in: Journal of Democracy, Vol. 6/No. 1, 65-78.

Reisch L. A./Hagen, K. (2011): Kann der Konsumentenwandel gelingen? Chancen und Grenzen einer verhaltensökonomisch basierten sozialen Regulierung, in: Heidbrink, L./Schmidt, I./Ahaus, B. (Hrsg.): Die Verantwortung des Konsumenten, Frankfurt a.M.: Campus, 221-245.

Richter, R./Furubotn, E. G. (2003): Neue Institutionenökonomik, Tübingen: Mohr Siebeck.

Schaller, R./Bachmann, R. (2004): Förderung und Unterstützung in der Freiwilligkeit durch Schweizer Unternehmen: Eine qualitative Befragung, in: Ammann, H./Bachmann, R./Schaller, R. (Hrsg.): Unternehmen unterstützen Freiwilligkeit. Freiwilligkeit, 1. Aufl. Zürich: Seismo, 30-69.

Schrader, U. (2003): Corporate Citizenship: Die Unternehmung als guter Bürger?, Berlin: Logos.

Stehr, N. (2007): Die Moralisierung der Märkte. Eine Gesellschaftstheorie, Frankfurt a.M.: Suhrkamp.

Steinmann, H./Löhr, A. (1994): Grundlagen der Unternehmensethik, 2. Auflage, Stuttgart: Schäffer-Poeschel.

Stolle, D./Hooghe, M./Micheletti, M. (2005): Politics in the Supermarket: Political Consumerismas a Form of Political Participation, in: International Political Science Review, Vol. 26/No. 3, 245-269.

Suchanek, A. (2007): Ökonomische Ethik, 2. Auflage, Tübingen: Mohr Siebeck.

Ulrich, P. (1998): Integrative Wirtschaftsethik, 2. Auflage, Bern; Stuttgart; Wien: Haupt.

Voigt, S. (2009): Institutionenökonomik, München: Fink Verlag.

Wang, L./Howell, J. P./Hinrichs, K. T./Prieto, L. (2010): Organizational Citizenship Behavior: The Role of Value/Identity-Based Motivation, in: Journal of Leadership \& Organizational Studies, Vol. 18/No. 1, 14-24.

Watrin, C. (1991): Ordnungssysteme für innerstaatliche wirtschaftliche Prozesse, in: Korff et al. (1999): Handbuch der Wirtschaftsethik 2. Ethik wirtschaftlicher Ordnungen, Gütersloh: Gütersloher Verlagshaus, 216-261.

Wildt, M. (1995): Am Beginn der Konsumgesellschaft. Mangelerfahrung, Lebenshaltung, Wohlstandshoffnung in Westdeutschland in den fünfziger Jahren, Hamburg: ErgebnisseVerlag.

Yoon, Y./Gürhan-Canli, Z./Schwar, N. (2006): The Effect of Corporate Social Responsibility (CSR) Activities on Companies With Bad Reputations, in: Journal of Consumer Psychology, Vol. 16/No. 4, 377-390. 